Supporting Information

\title{
Synthesis of meso-Arylsulfanyl- and Alkylsulfanyl-Substituted Porphyrins via Palladium-Mediated C-S Bond Formation
}

Guang-Yao Gao, Andrew J. Colvin, Ying Chen and X. Peter Zhang*

Department of Chemistry, University of Tennessee, Knoxville, TN 37996

Table of Contents

${ }^{1}$ H-NMR Spectrum of 10-(Naphthalen-1-ylsulfanyl)-5,15-diphenylporphyrin $\quad$ S2

$\begin{array}{ll}{ }^{1} H \text { H-NMR Spectrum of 10-(o-Tolylsulfanyl)-5,15-diphenylporphyrin } & \text { S3 }\end{array}$

'H-NMR Spectrum of 10-(4'-Methoxyphenylsulfanyl)-5,15-diphenylporphyrin $\quad$ S4

${ }^{1}$ H-NMR Spectrum of 10-(4'-Chlorophenylsulfanyl)-5,15-diphenylporphyrin $\quad$ S5

${ }^{1}$ H-NMR Spectrum of 10-Benzylsulfanyl-5,15-diphenylporphyrin $\quad$ S6

$\begin{array}{ll}{ }^{1} \mathrm{H}-\mathrm{NMR} \text { Spectrum of 10-Octylsulfanyl-5,15-diphenylporphyrin } & \text { S7 }\end{array}$

$\begin{array}{ll}{ }^{1} \mathrm{H}-\mathrm{NMR} \text { Spectrum of 10-Cyclohexylsulfanyl-5,15-diphenylporphyrin } & \text { S8 }\end{array}$

${ }^{1}$ H-NMR Spectrum of 10-(4'-Acetamidobenezensulfanyl)-5,15-diphenylporphyrin $\quad$ S9

${ }^{1} \mathrm{H}$-NMR Spectrum of 10-(Benzothiazol-2-ylsulfanyl)-5,15-diphenylporphyrin $\quad$ S10

${ }^{1}$ H-NMR Spectrum of 10-(Propane-3-thiol)-sulfanyl-5,15-diphenylporphyrin $\quad$ S11

${ }^{1} \mathrm{H}$-NMR Spectrum of 10-Phenylselanyl-5,15-diphenylporphyrin $\quad$ S12

${ }^{1} \mathrm{H}$-NMR Spectrum of 5,15-Bis(2'-methylphenylsulfanyl)-10,20-diphenyl-porphyrin $\quad$ S13

${ }^{1} \mathrm{H}$-NMR Spectrum of 5,15-Bis(4'-methoxyphenylsulfanyl)-10,20-diphenylporphyrin $\quad$ S14

${ }^{1} \mathrm{H}$-NMR Spectrum of 5,15-Bis(4'-chlorophenylsulfanyl)-10,20-diphenylporphyrin $\quad$ S15

${ }^{1}$ H-NMR Spectrum of 5,15-Bis(propylsulfanyl)-10,20-diphenylporphyrin $\quad$ S16

${ }^{1}$ H-NMR Spectrum of 5,15-Bis(cyclohexylsulfanyl)-10,20-diphenylporphyrin $\quad$ S17

${ }^{1}$ H-NMR Spectrum of [10-(2'-Methylphenylsulfanyl)-5,15-diphenylporphinato]Zinc(II) $\quad$ S18

${ }^{1}$ H-NMR Spectrum of [10-(4'-Methoxyphenylsulfanyl)-5,15-diphenylporphinato]Zinc(II) $\quad$ S19

${ }^{1}$ H-NMR Spectrum of [10-(4'-Chlorophenylsulfanyl)-5,15-diphenylporphinato]Zinc(II) $\quad$ S20

${ }^{1}$ H-NMR Spectrum of [10-Propylsulfanyl-5,15-diphenylporphinato]Zinc(II) $\quad$ S21

${ }^{1}$ H-NMR Spectrum of [10-Cyclohexylsulfanyl-5,15-diphenylporphinato]Zinc(II) $\quad$ S22

${ }^{1}$ H-NMR Spectrum of [10-(4'-Acetamidobenezensulfanyl)-5,15-diphenylporphinato]Zinc(II) $\quad$ S23

${ }^{1}$ H-NMR Spectrum of [10-(Benzothiazol-2-ylsulfanyl)-5,15-diphenylporphinato]Zinc(II) $\quad$ S24 

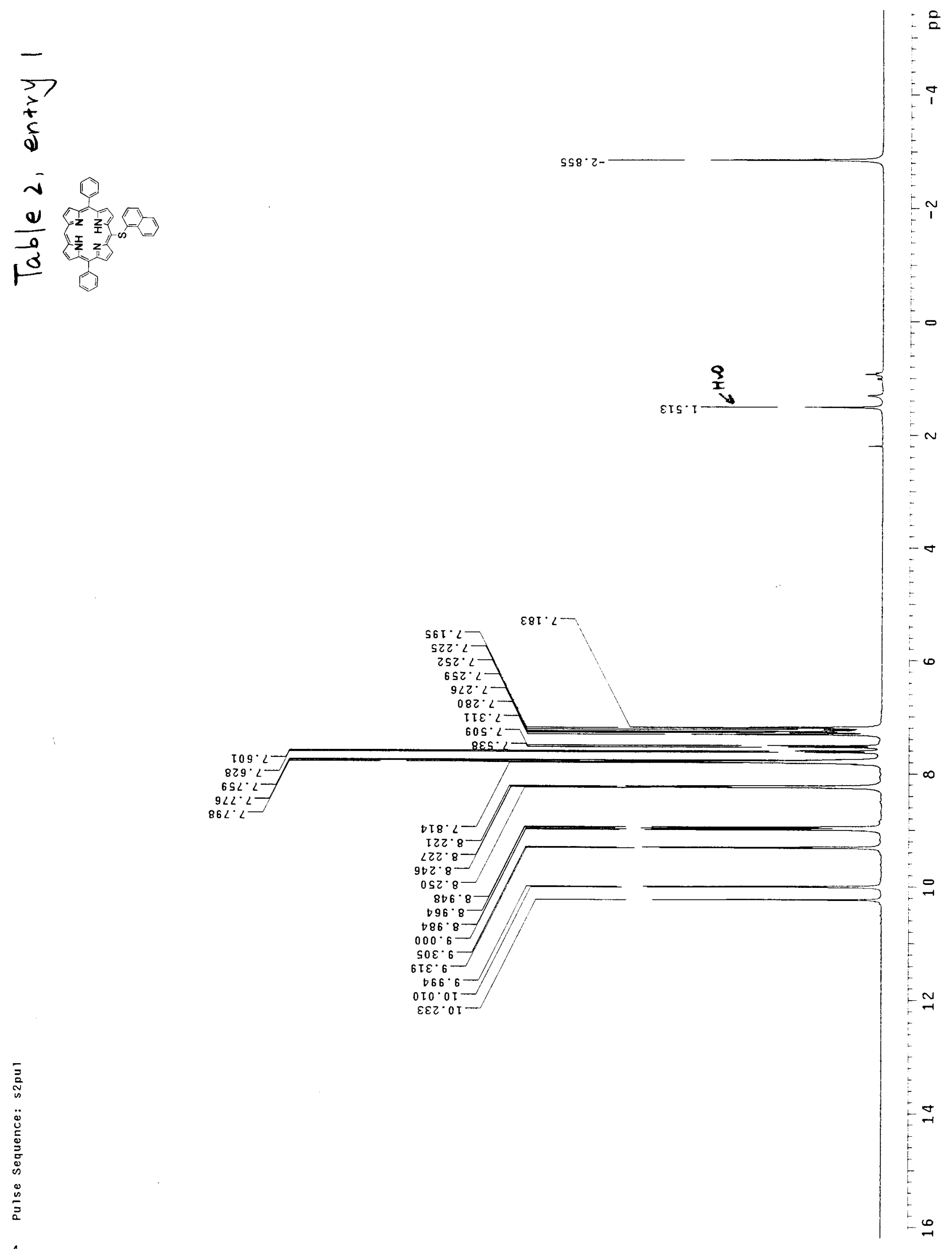
$\frac{\text { रे }}{5}$

a)

i

专
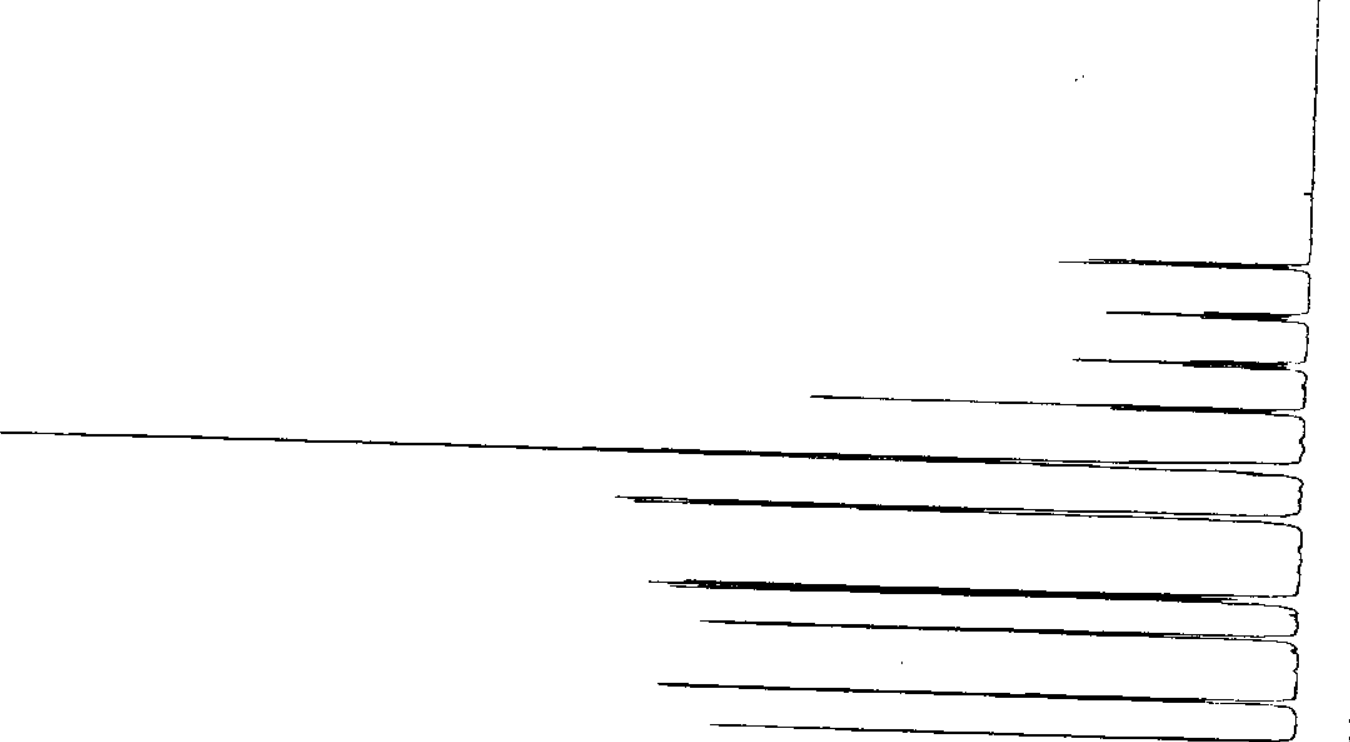

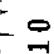

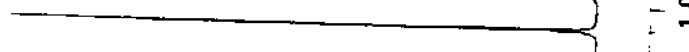




$$
\frac{1}{4}
$$
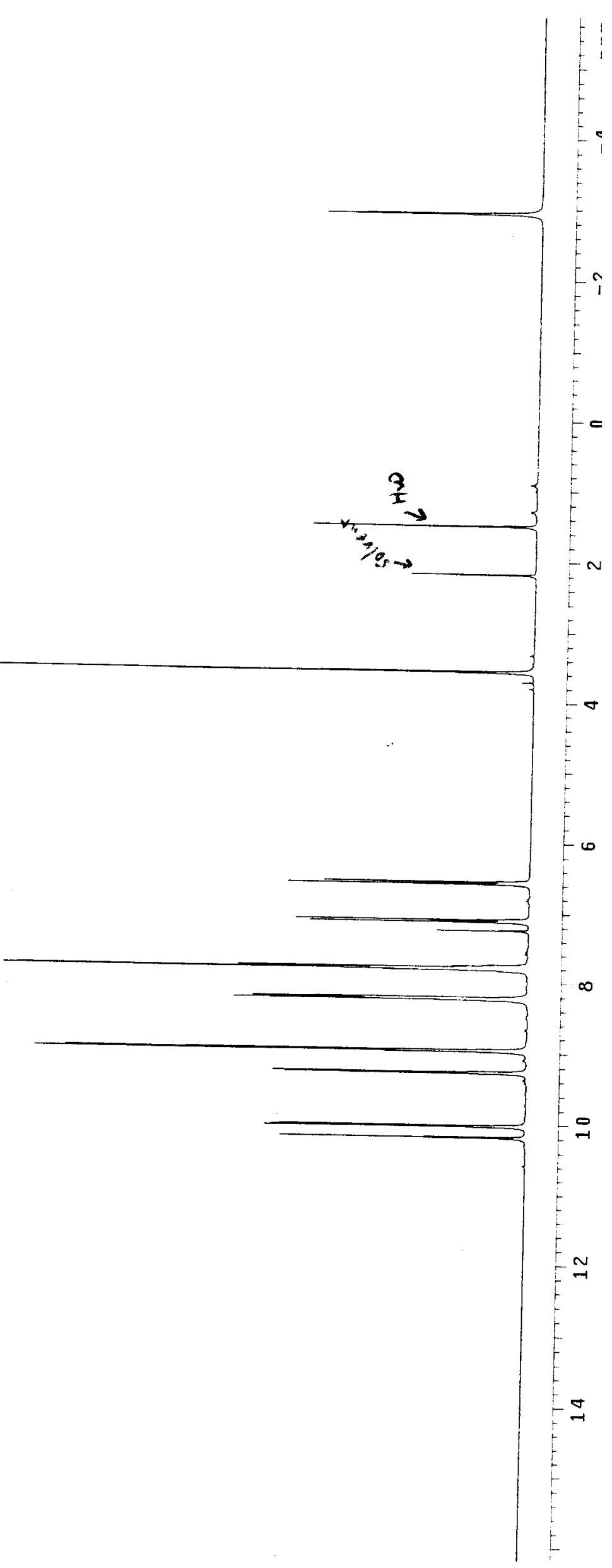


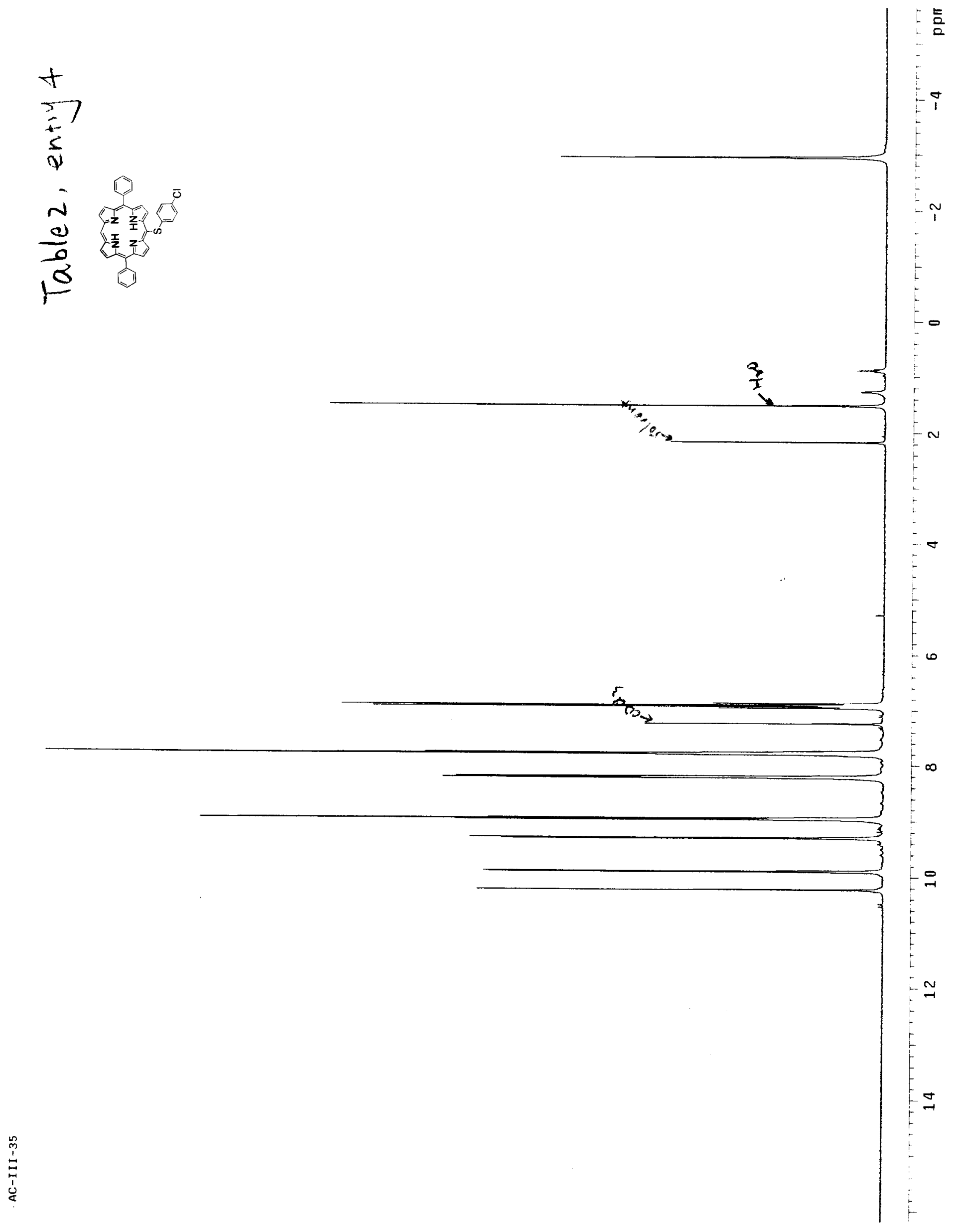



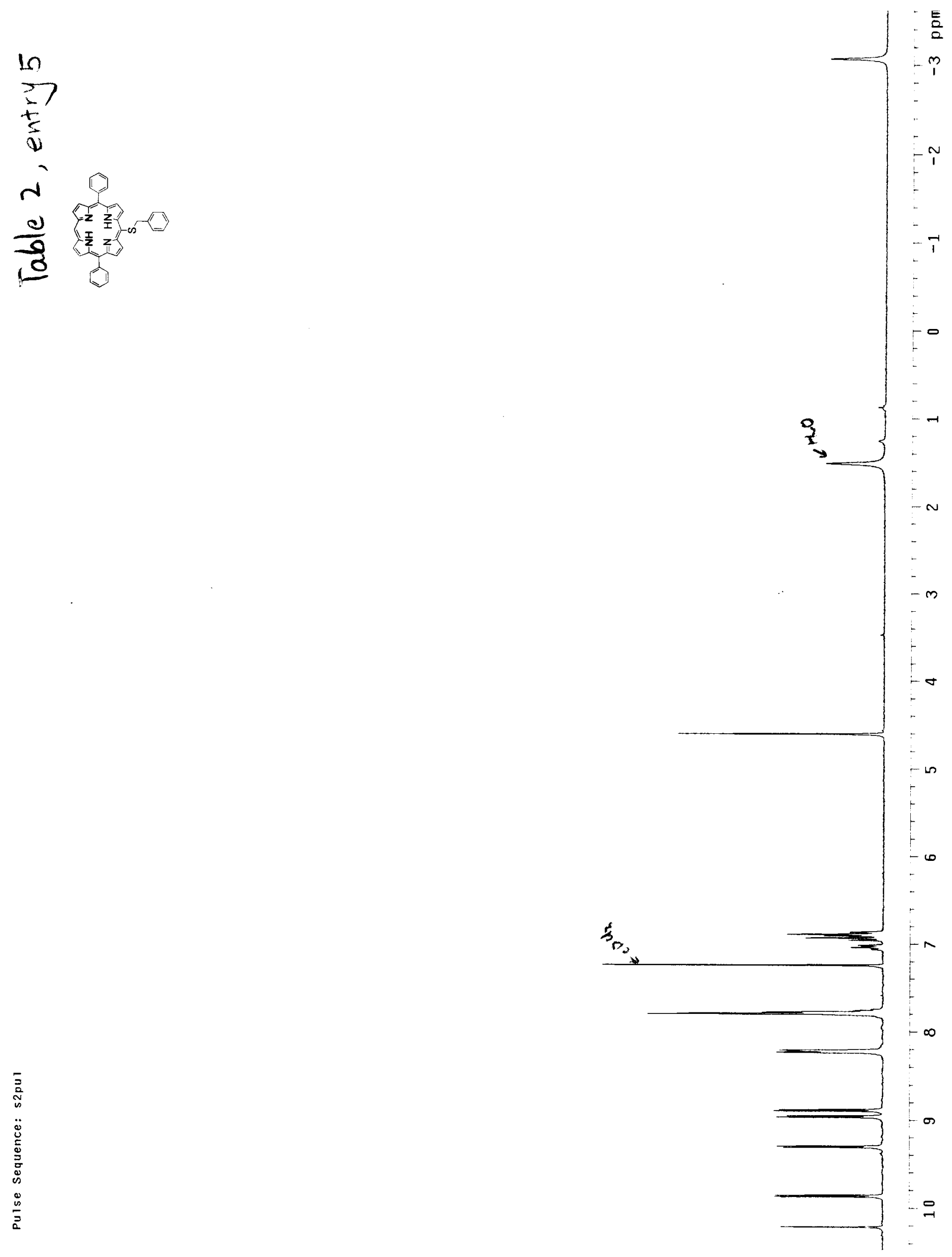

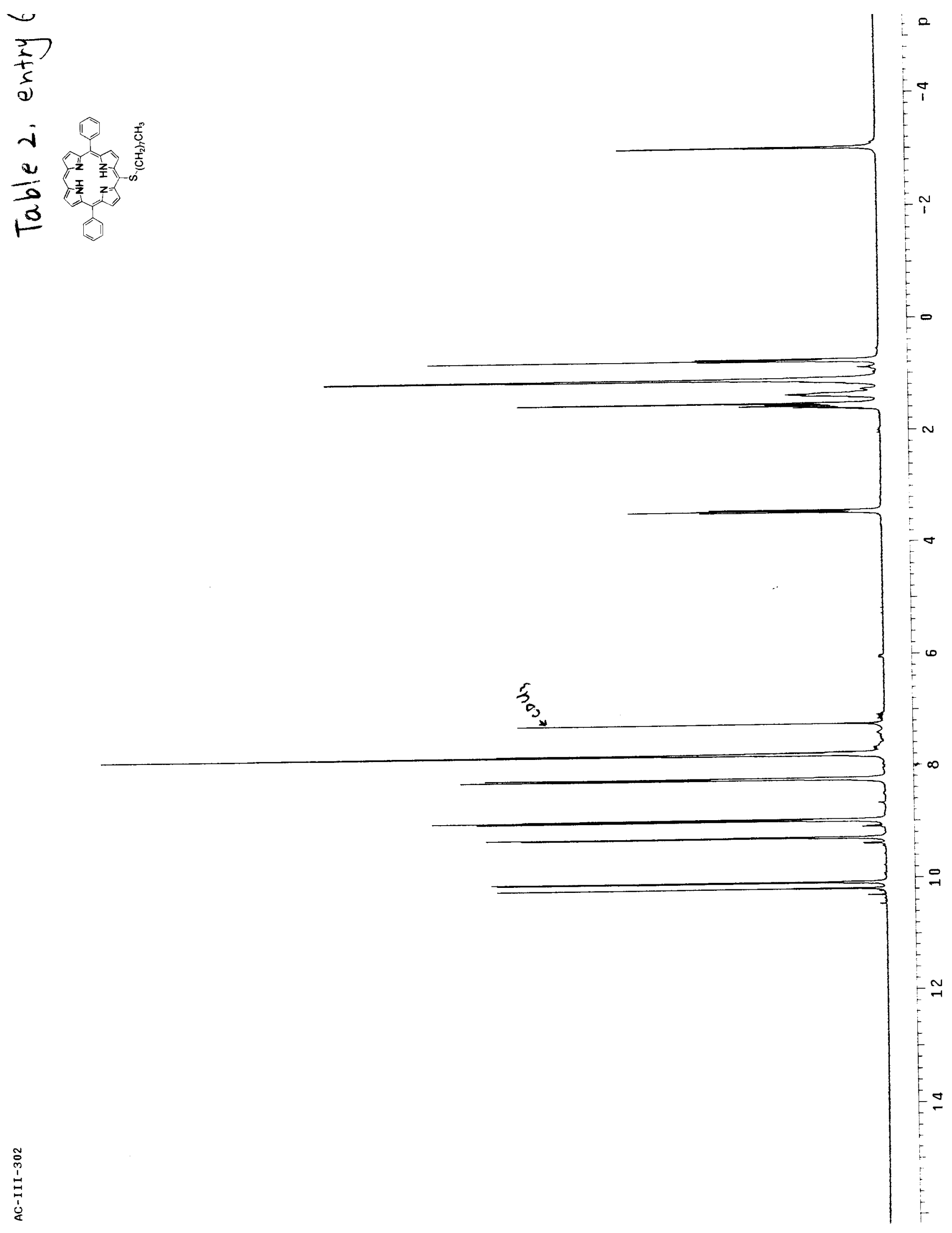
5
5
5
4
0
0
0

$x$
1
1
1
$y$

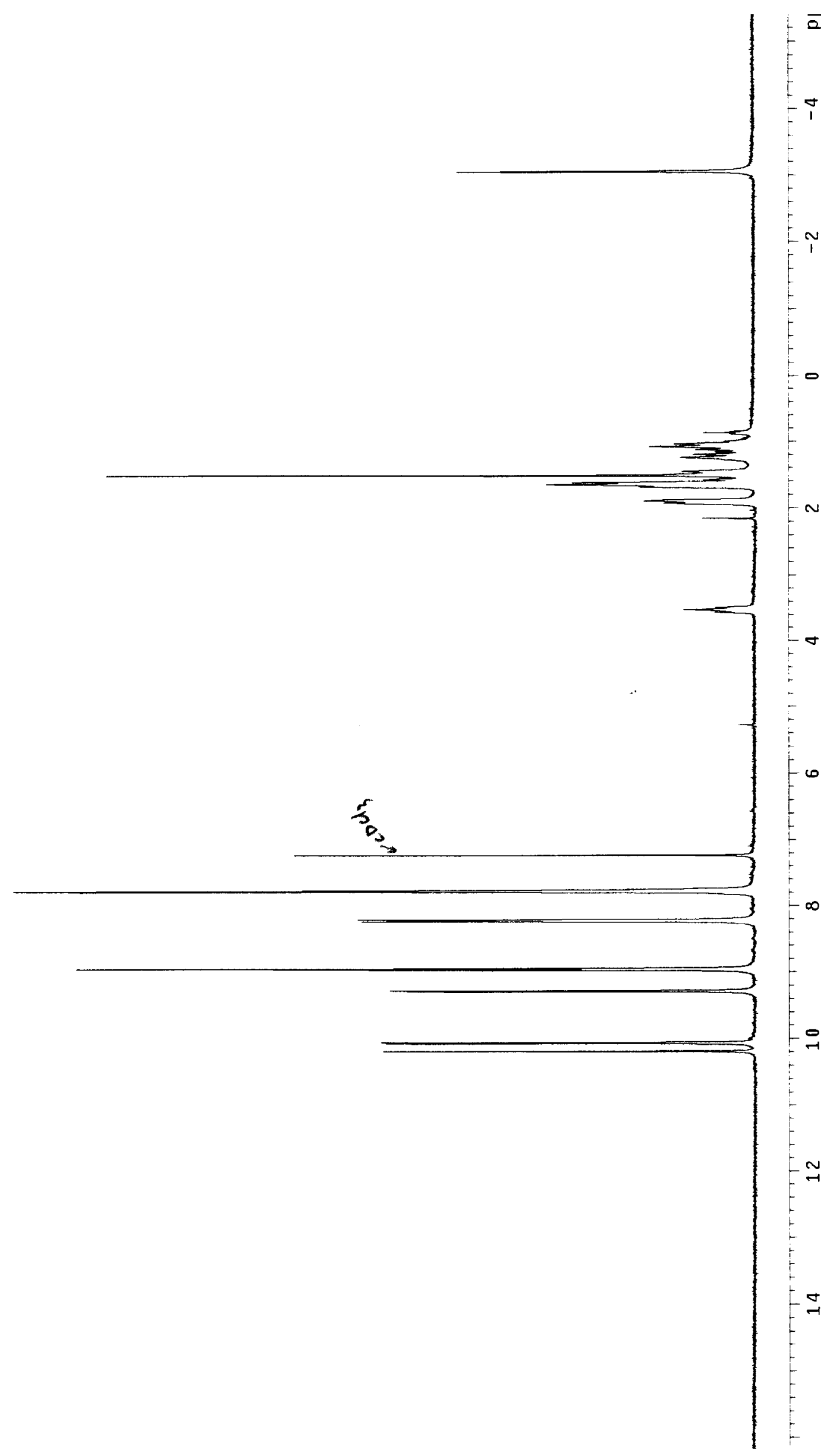



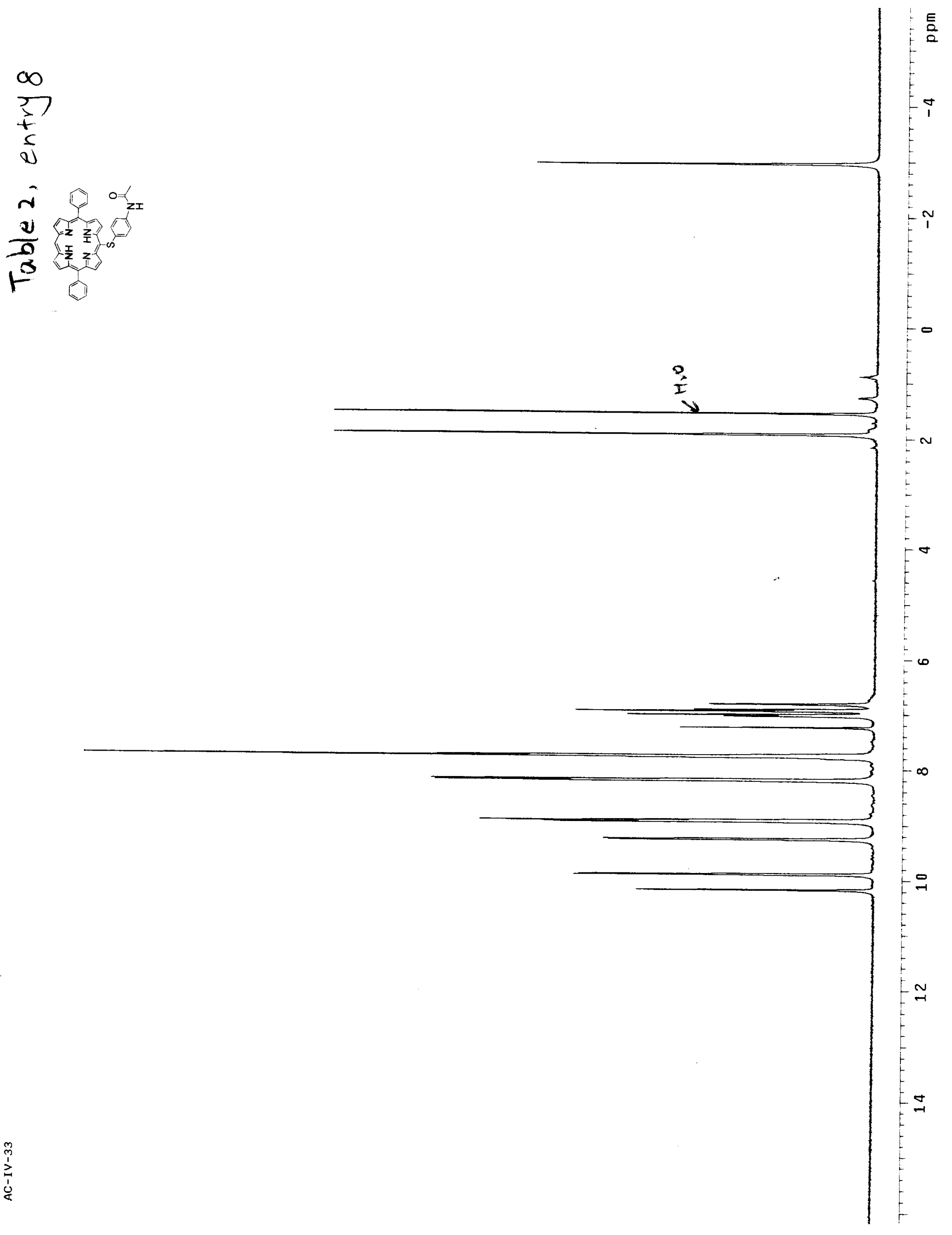
$\frac{7}{2}$

$\frac{1}{0}$ (1)

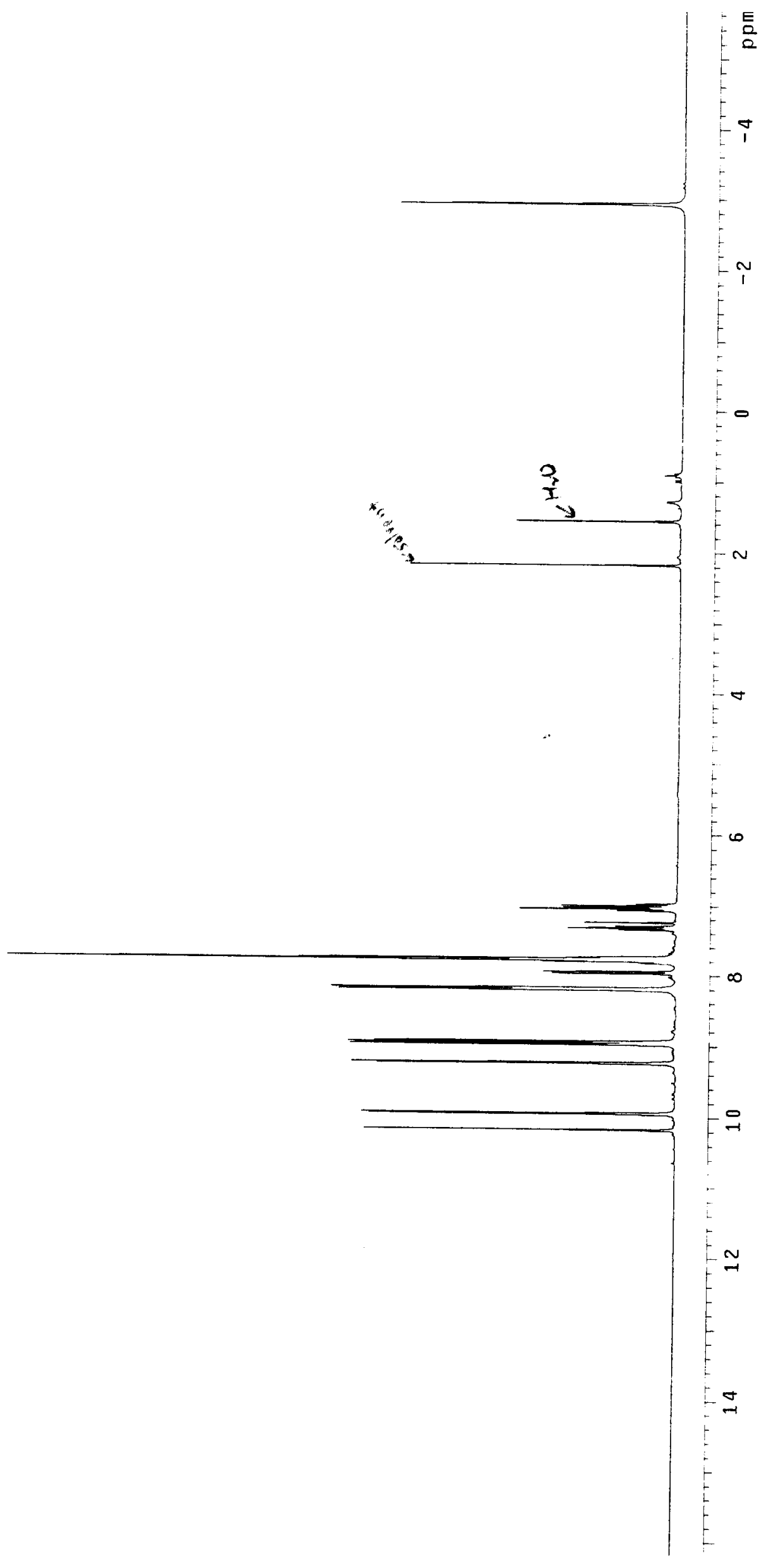


$\frac{3}{5} 1$

Q

त औ

$\frac{9}{0} 10$

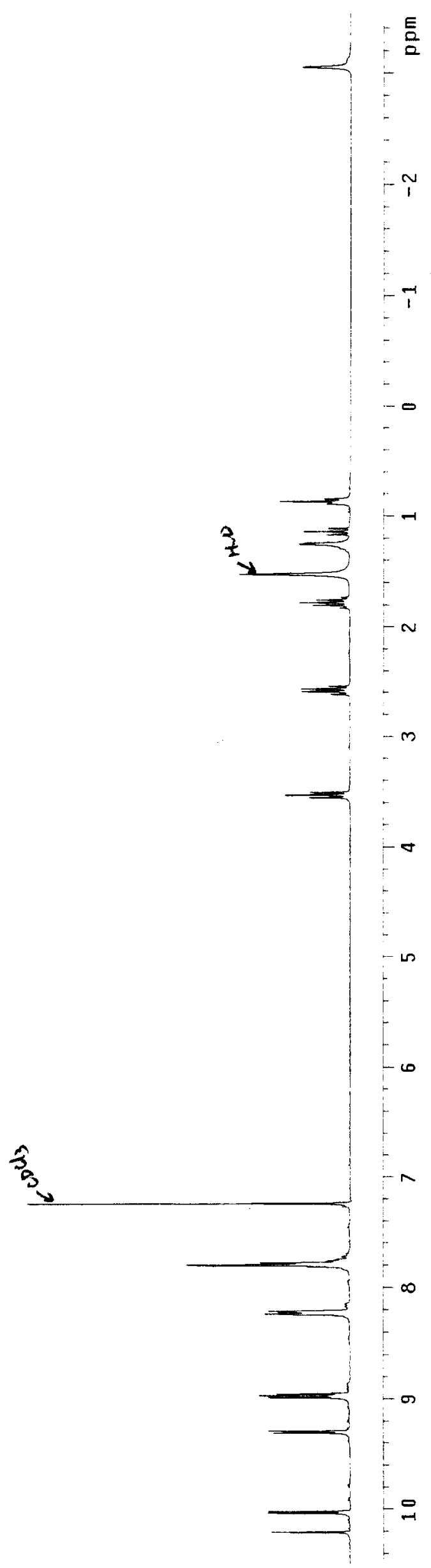

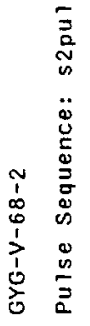



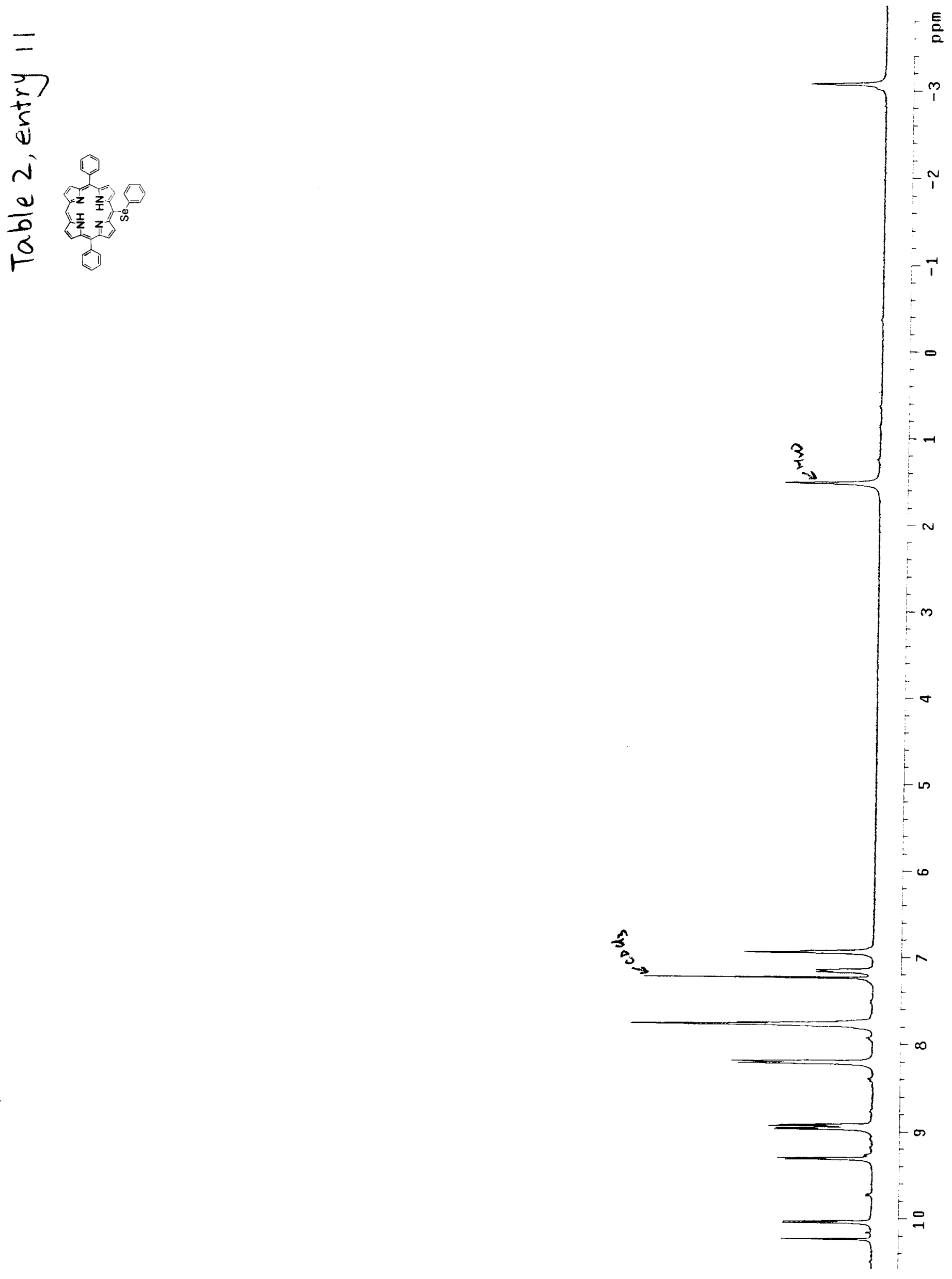

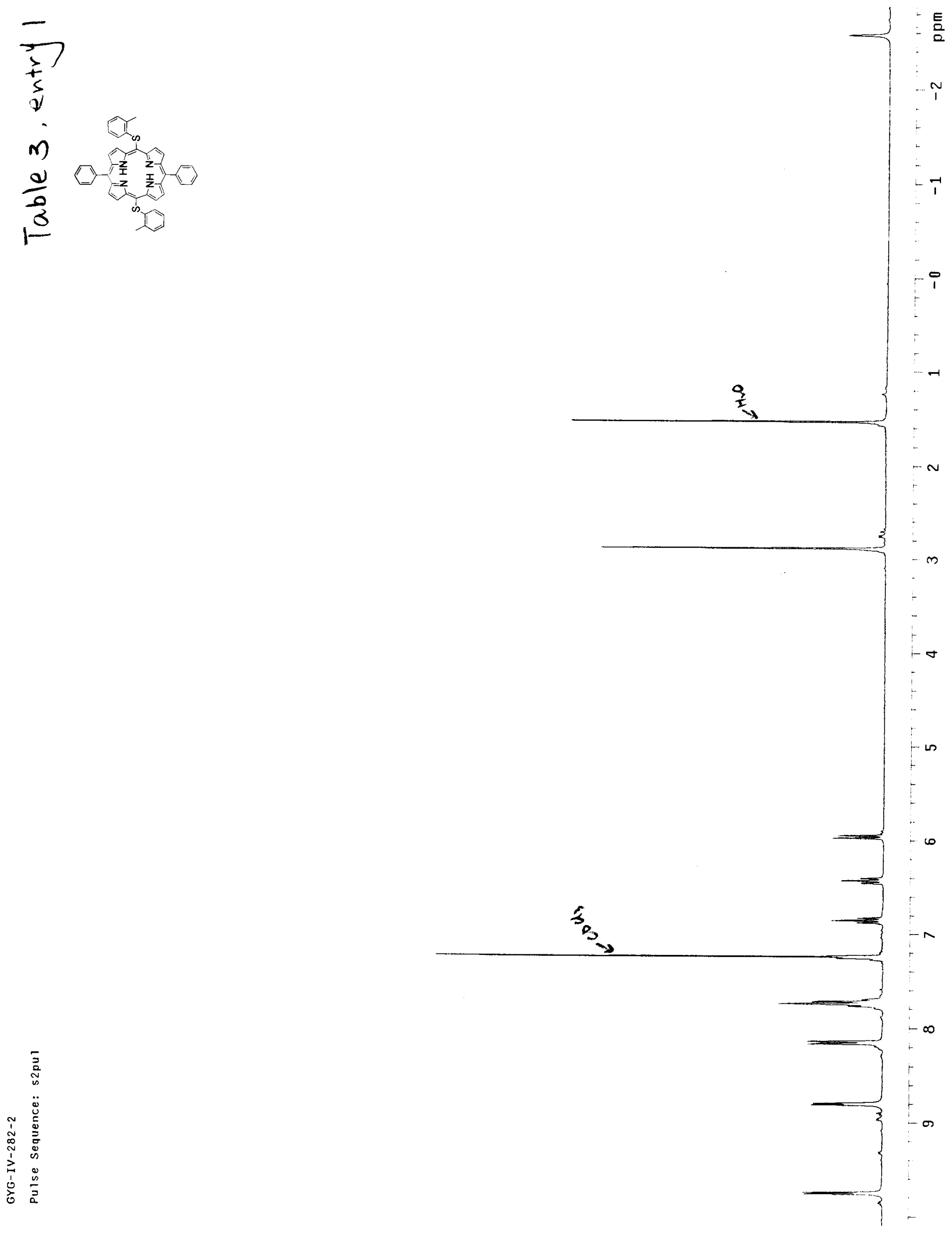

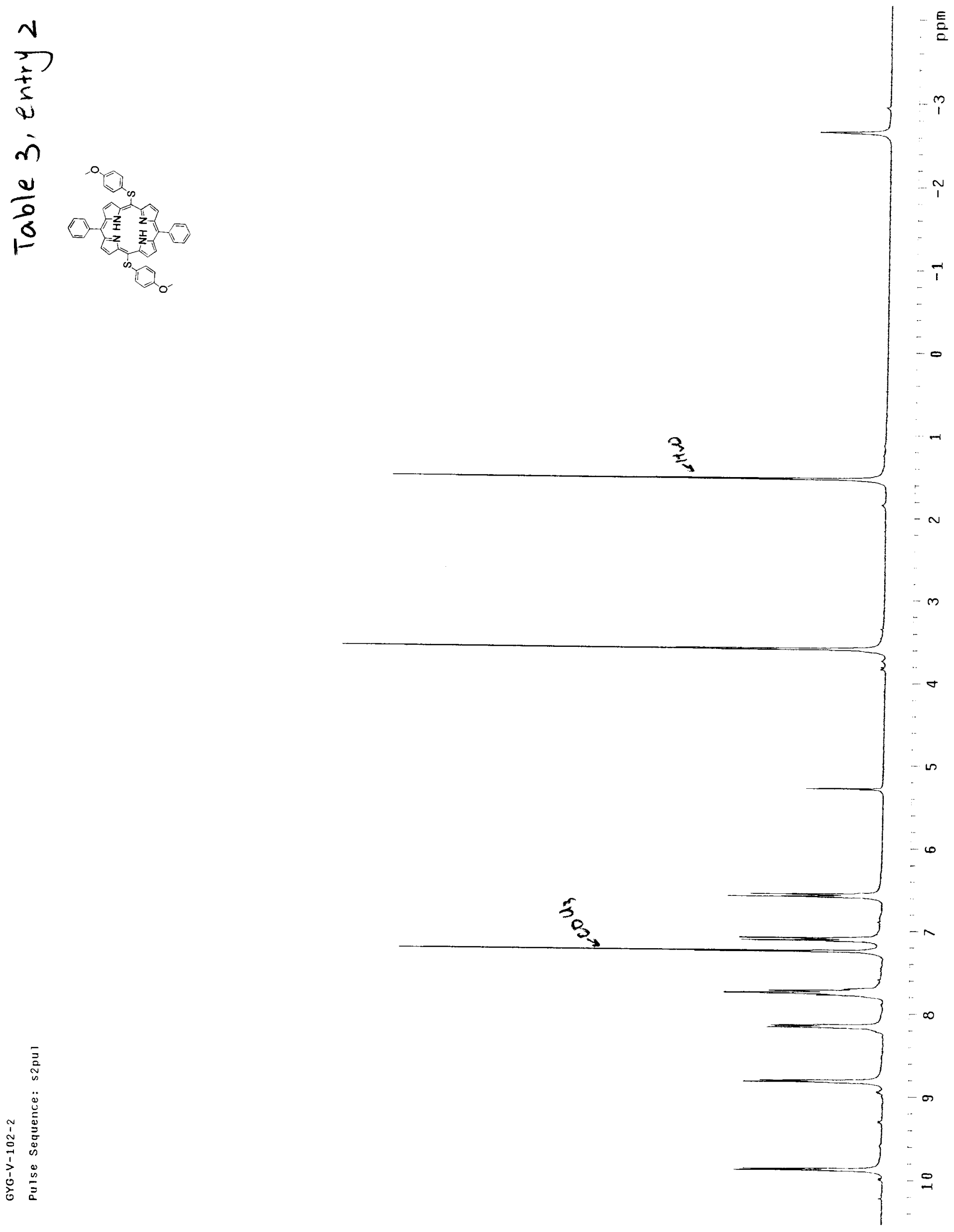

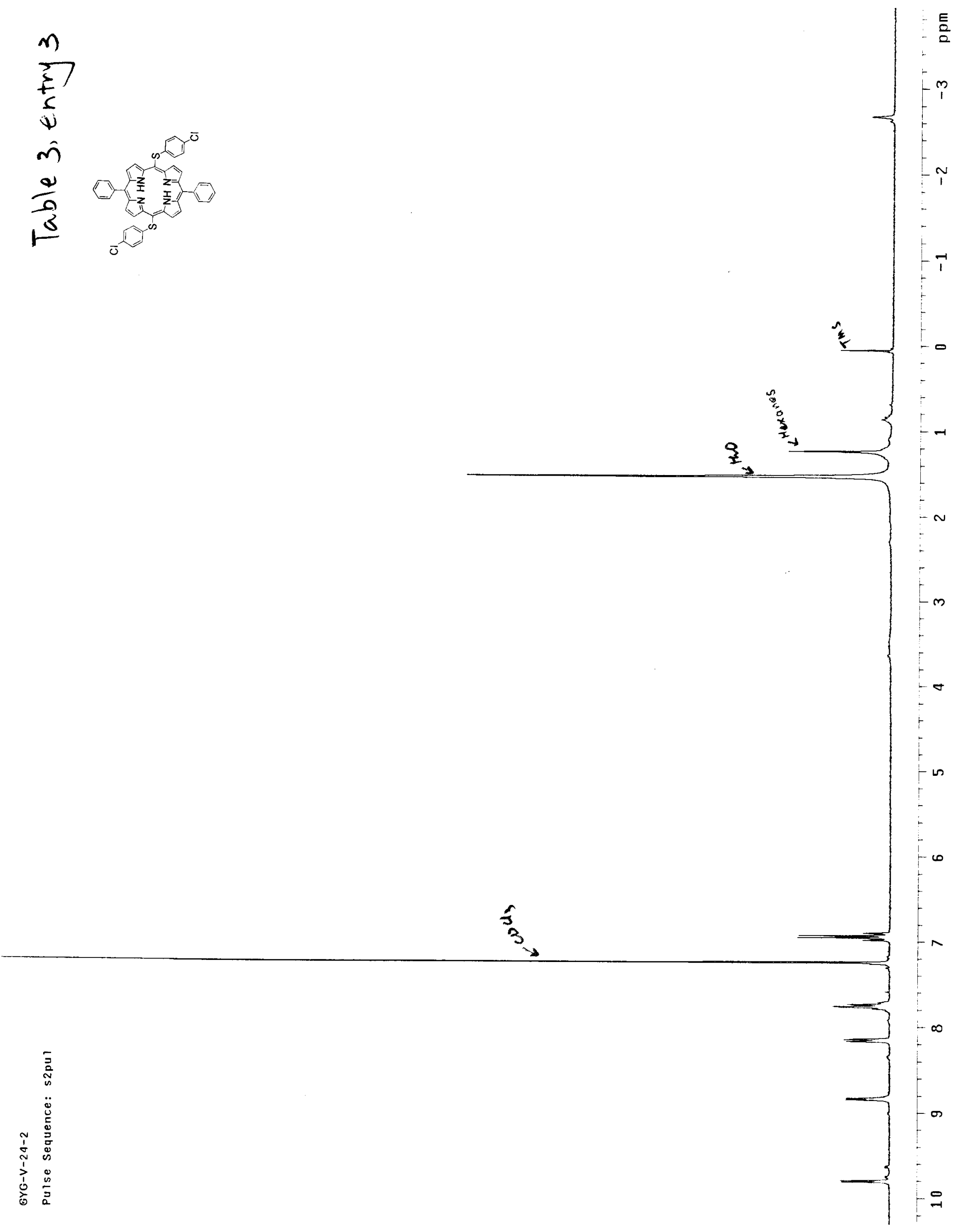


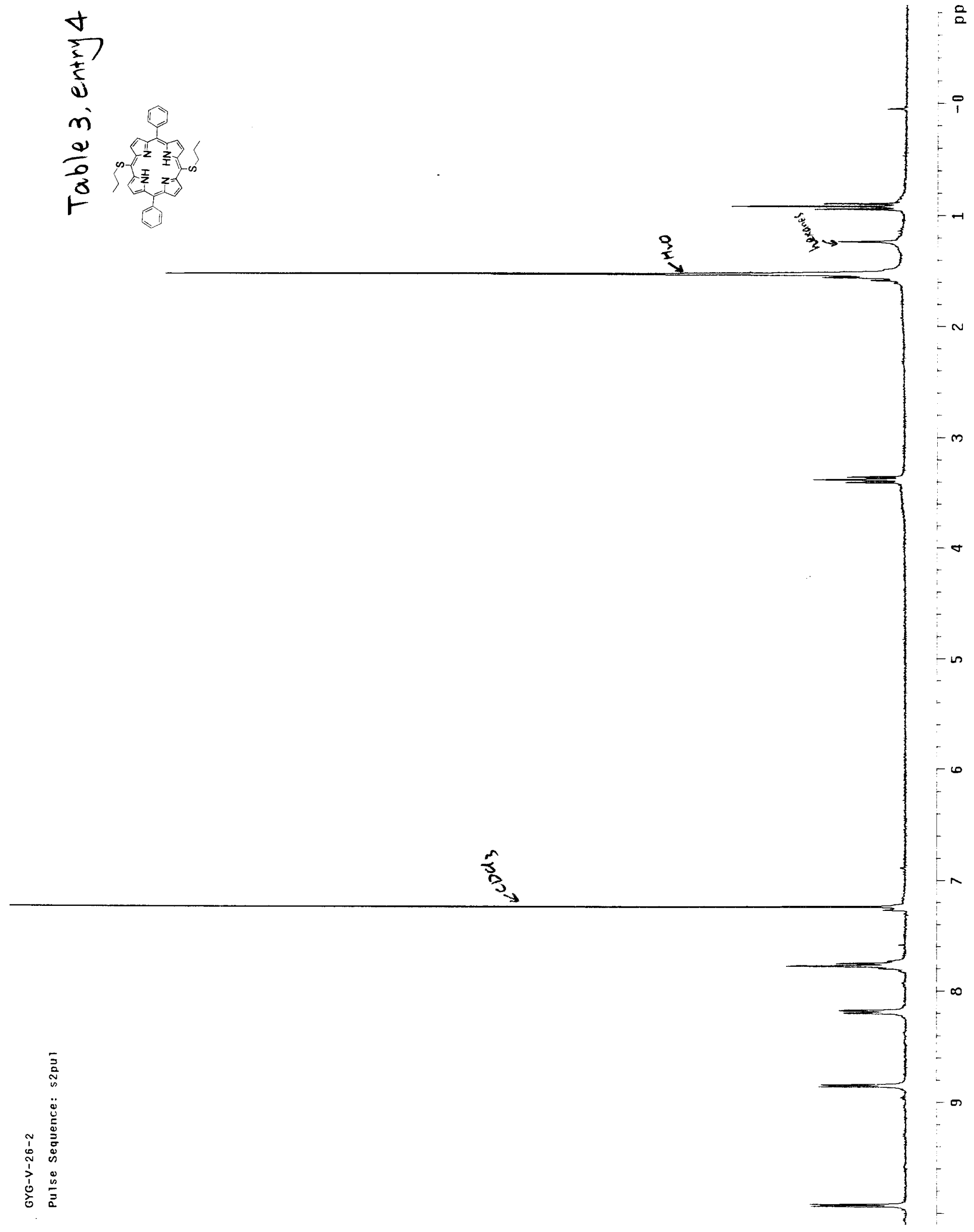



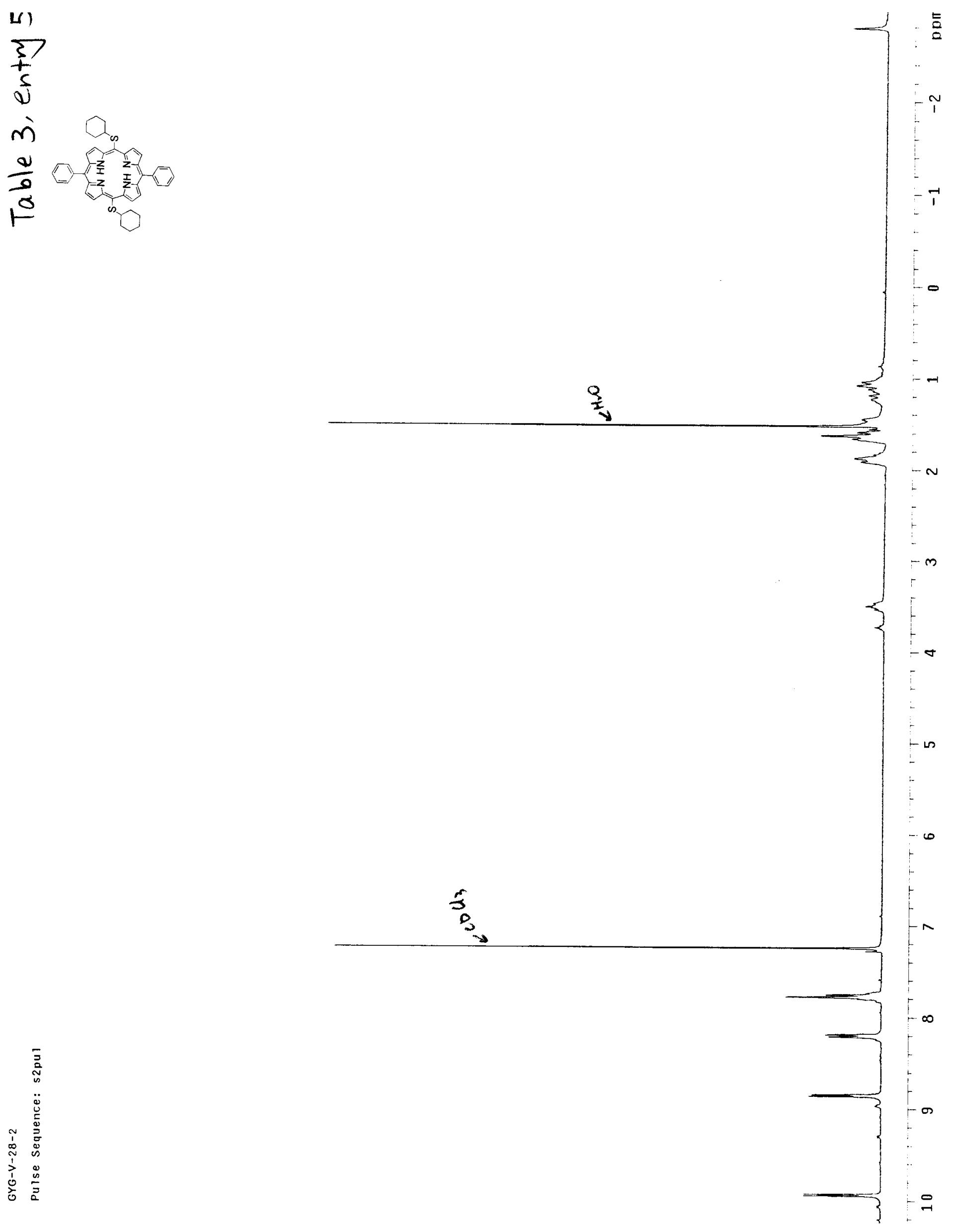

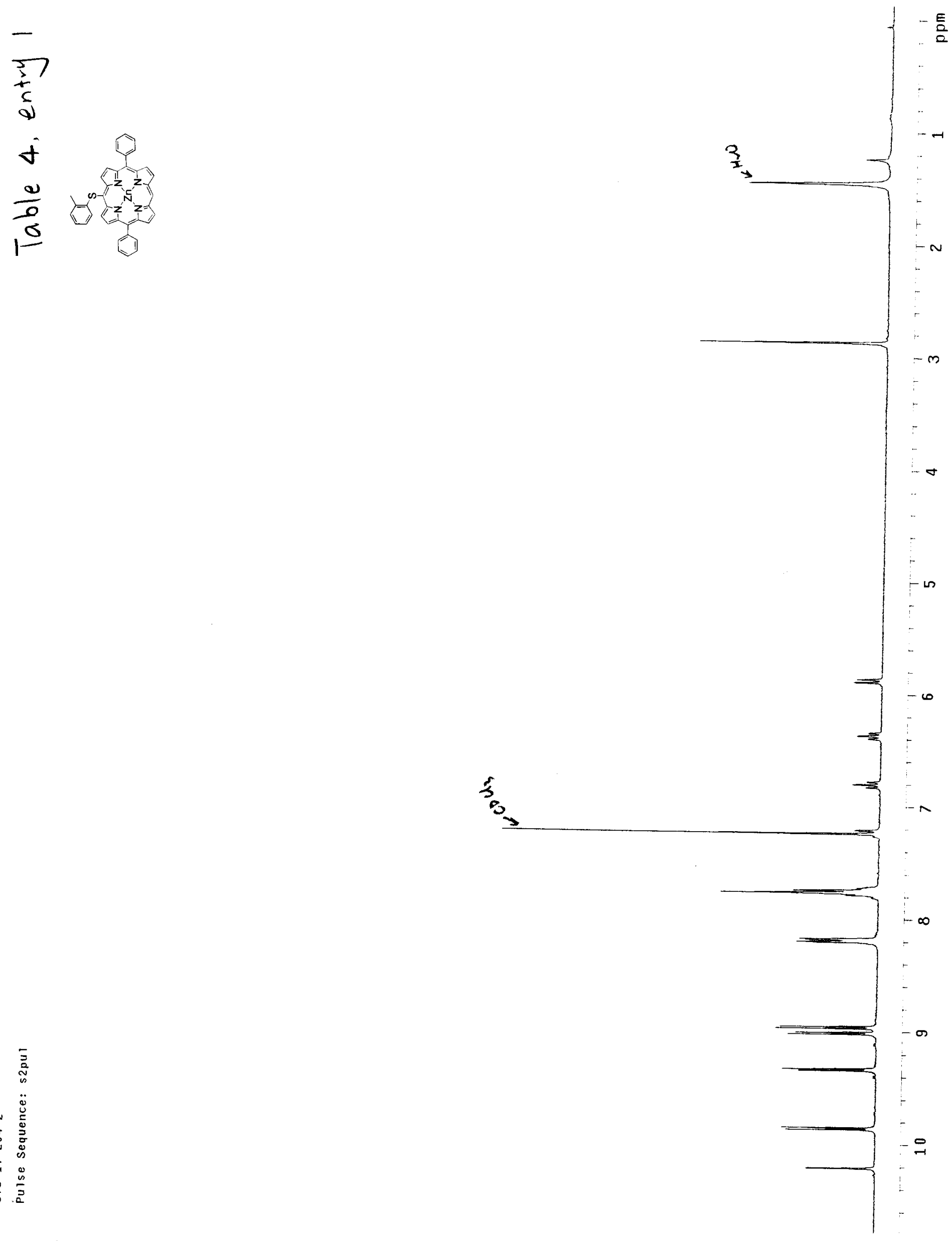


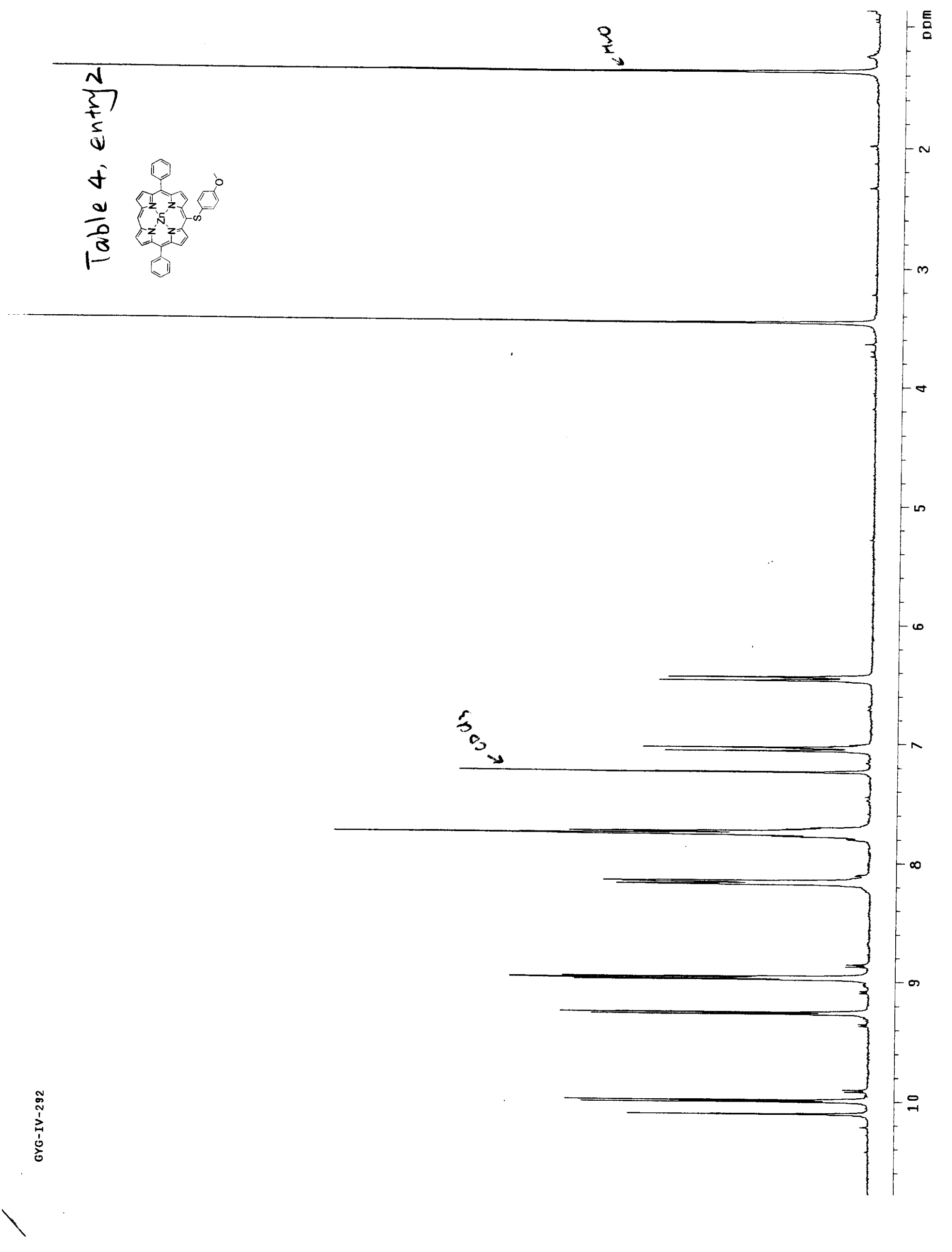



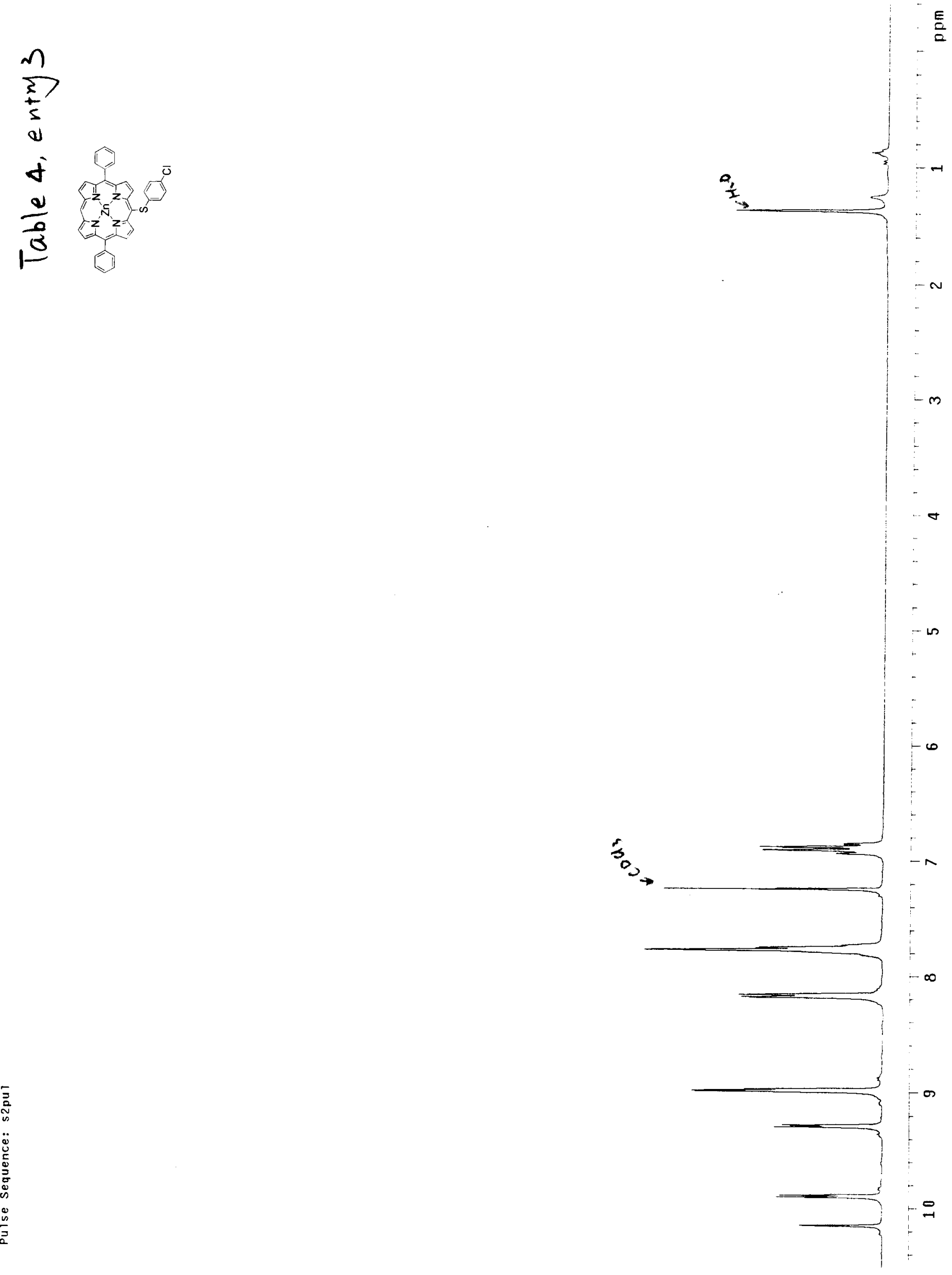

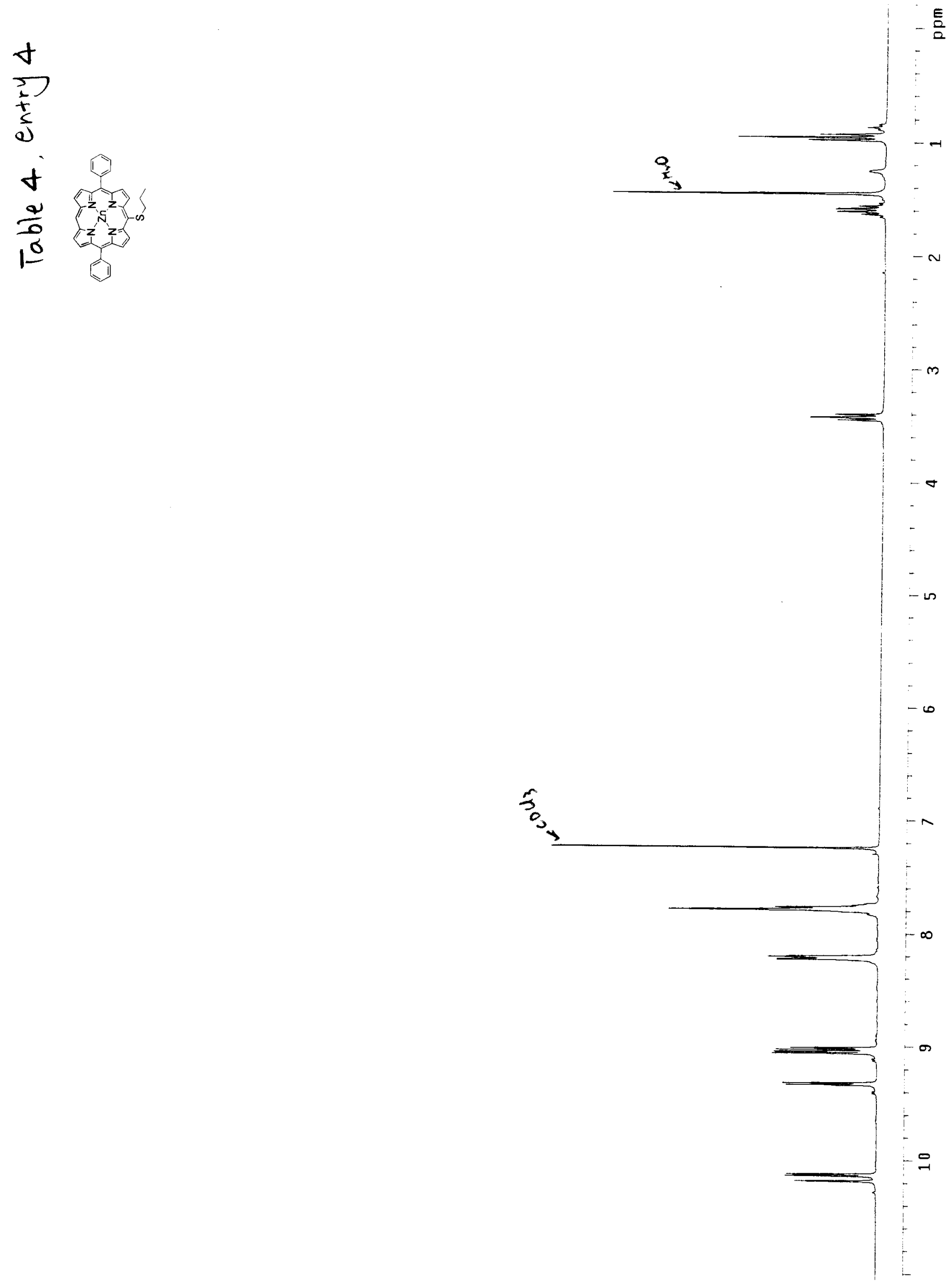


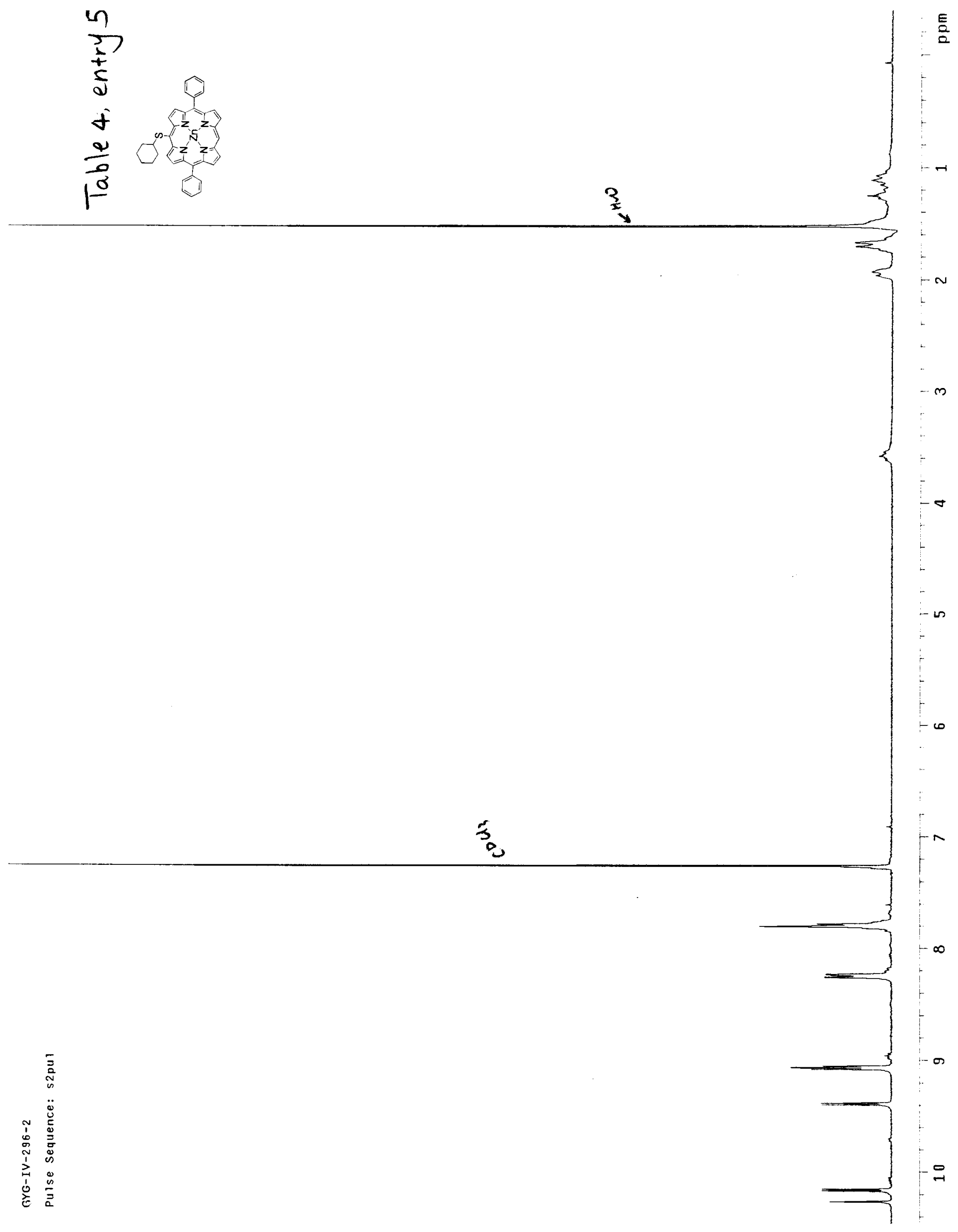



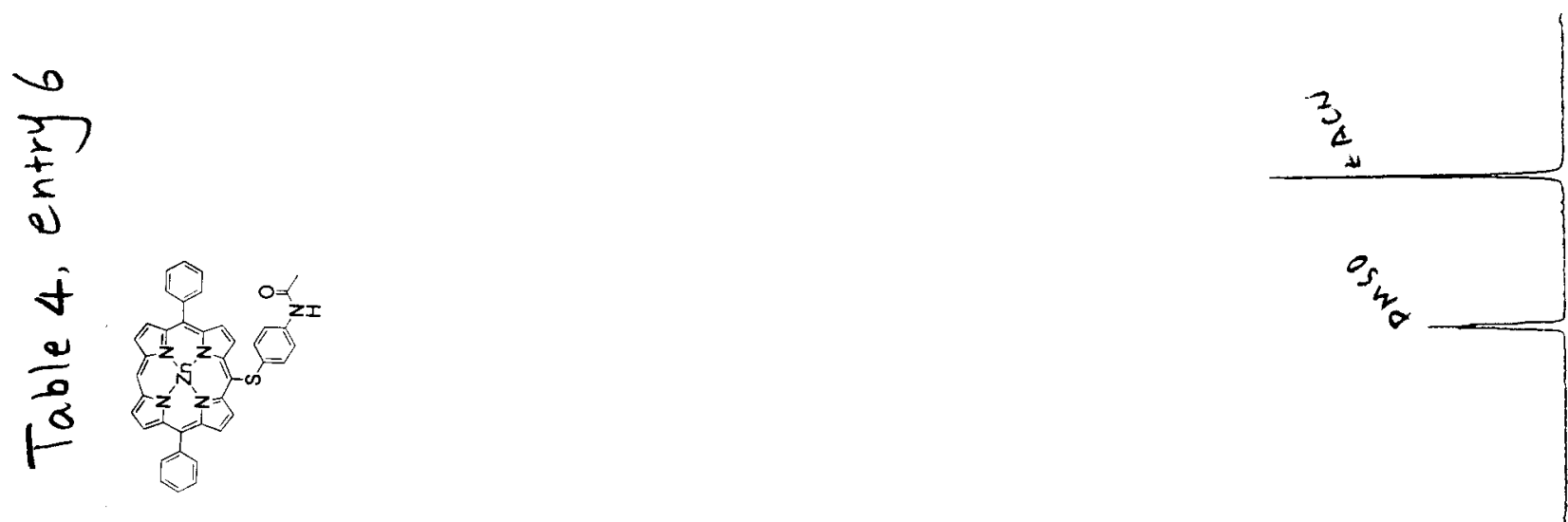

$\frac{a}{2}$

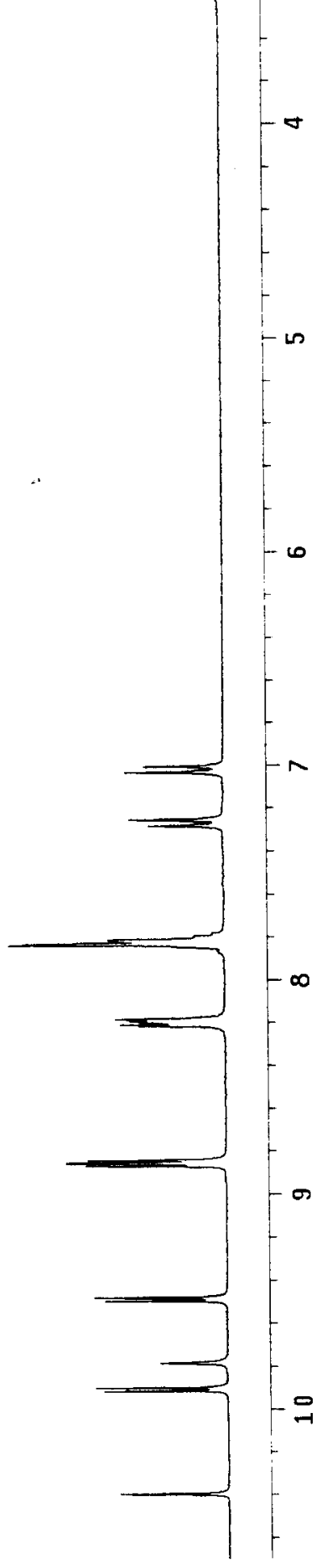




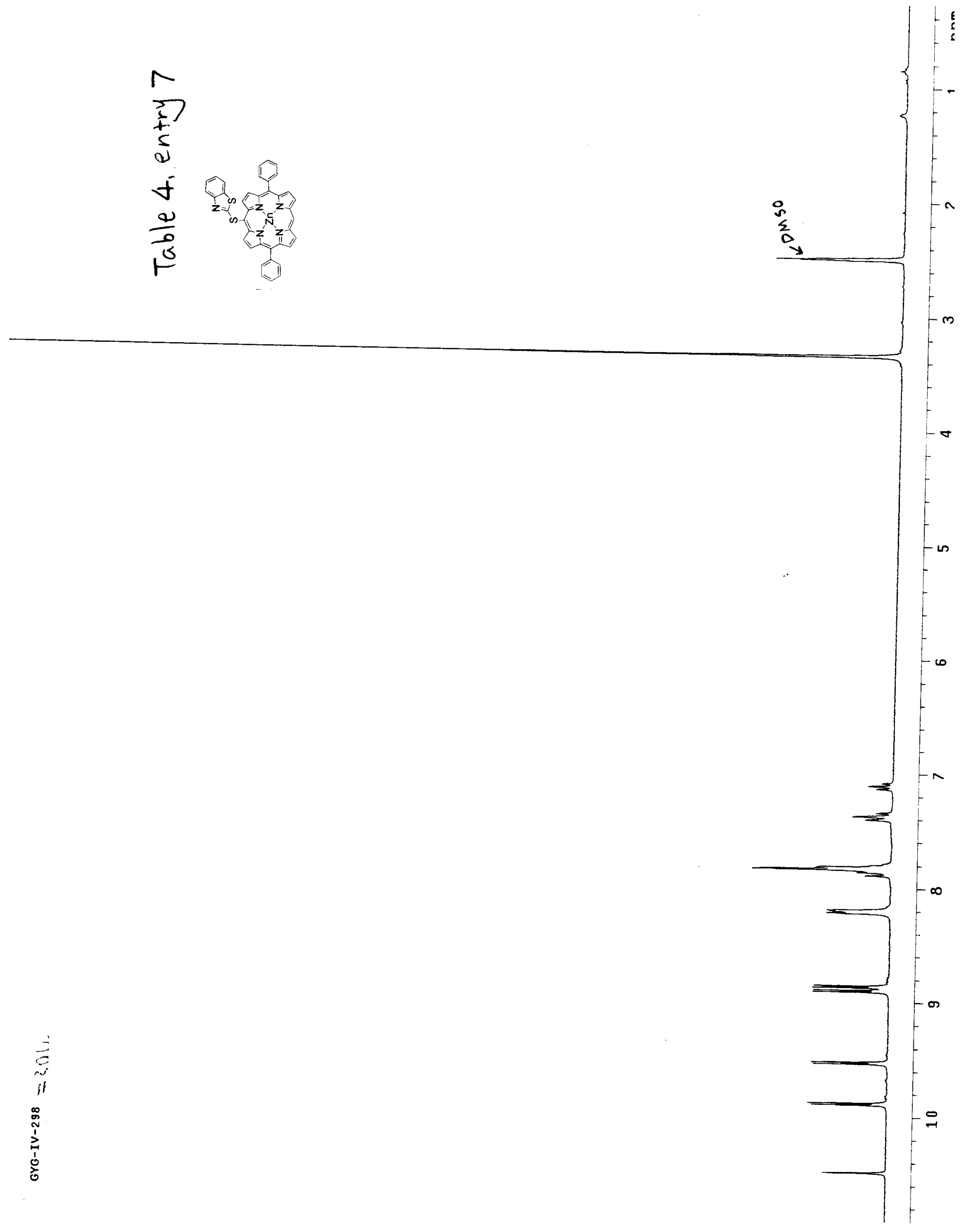

